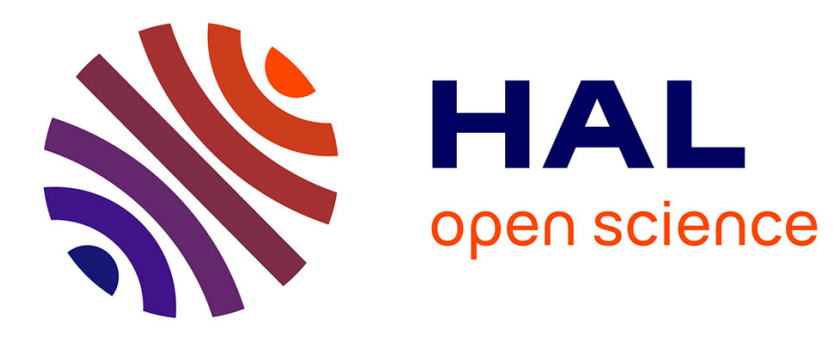

\title{
Paramutation phenomena in non-vertebrate animals
}

Stéphane Ronsseray

\section{To cite this version:}

Stéphane Ronsseray. Paramutation phenomena in non-vertebrate animals. Seminars in Cell and Developmental Biology, 2015, 44, pp.39-46. 10.1016/j.semcdb.2015.08.009 . hal-01204543

\section{HAL Id: hal-01204543 https: / hal.sorbonne-universite.fr/hal-01204543}

Submitted on 28 Sep 2015

HAL is a multi-disciplinary open access archive for the deposit and dissemination of scientific research documents, whether they are published or not. The documents may come from teaching and research institutions in France or abroad, or from public or private research centers.
L'archive ouverte pluridisciplinaire $\mathbf{H A L}$, est destinée au dépôt et à la diffusion de documents scientifiques de niveau recherche, publiés ou non, émanant des établissements d'enseignement et de recherche français ou étrangers, des laboratoires publics ou privés. 


\section{Paramutation phenomena in non-vertebrate animals}

Stéphane RONSSERAY ${ }^{1,2}$

${ }^{1}$ Sorbonne Universités, UPMC Univ Paris 06, Institut de Biologie Paris-Seine (IBPS), UMR 7622, Developmental Biology, 9 quai Saint-Bernard, F-75005 Paris, France

${ }^{2}$ CNRS, IBPS, UMR 7622, Developmental Biology, 9 quai Saint-Bernard, F-75005 Paris, France

stephane.ronsseray@upmc.fr 


\section{Abstract}

Paramutation was initially described in maize and was defined as an epigenetic interaction between two alleles of a locus, through which one allele induces a heritable modification of the other allele without modifying the DNA sequence [1,2]. Thus it implies that the paramutated allele conserves its new properties on the long term over generations even in the absence of the paramutagenic allele and that it turns paramutagenic itself, without undergoing any changes in the DNA sequence. Some epigenetic interactions have been described in two non-vertebrate animal models, which appear to exhibit similar properties. Both systems are linked to trans-generational transmission of non-coding small RNAs. In Drosophila melanogaster, paramutation is correlated with transmission of PIWIInteracting RNAs (piRNAs), a class of small non-coding RNAs that repress mobile DNA in the germline. A tandem repeated transgenic locus producing abundant ovarian piRNAs can activate piRNA production and associated homology-dependent silencing at a locus that was previously stably devoid of such capacities. The newly converted locus is then perfectly stable in absence of the inducer locus (>100 generations) and becomes fully paramutagenic. In Caenorhabditis elegans, paramutation is correlated with transmission of siRNAs, which are produced by transgenes targeted by piRNAs in the germline. Indeed, a transgenic locus, targeted by the piRNA machinery, produces siRNAs that can induce silencing of homologous transgenes, which can be further transmitted in a repressed state over generations despite the absence of the inducer transgenic locus. As in fly, the paramutated locus can become fully paramutagenic, and paramutation can be mediated by cytoplasmic inheritance without transmission of the paramutagenic locus itself. Nevertheless, in contrast to flies where the induction is only maternally inherited, both parents can transmit it in worms. In addition, a reciprocal phenomenon - (from off toward on) - appears to be also possible in worms as some activated transgenes can reactivate silent transgenes in the germline, and this modification can also be transmitted to next generations, even so it appears to be only partially stable. Thus, in a given system, opposite paramutation-like phenomena could exist, mediated by antagonist active pathways. As in plants, paramutation in flies and worms correlates with chromatin structure modification of the paramutated locus. In flies, inheritance of small RNAs from one generation to the next transmits a memory mainly targeting loci for repression whereas in worms, small RNAs can target loci either for repression or expression. Nevertheless, in the two species, paramutation can play an important role in the epigenome establishment. 


\section{1- Introduction}

Various strategies are used to recognize and repress mobile or foreign DNAs in genomes and to epigenetically transfer silencing memory over generations. In fly, transgenerational information seems to ensure predominantly gene repression. Indeed, systems based on the principle of genomic traps have been established in order to identify and further repress Transposable Elements (TEs). The Drosophila melanogaster genome contains about 140 discrete loci composed of TE fragments which undergo non-canonical transcription. This results in the inactivation of the transcript of the locus itself and of all the homologous transcripts produced by the genome [3-6]. Therefore, this system based on the PIWI-Interacting RNAs (piRNAs) results in repression of all TE copies in the genome if at least one copy has inserted into a piRNA-producing locus. Euchromatic TE repression occurs at both the transcriptional and post-transcriptional levels [7-10]. Thus, when a TE copy is active, it will move and insert into a piRNA-producing locus and this "piRNA locus" copy will progressively establish repression of the entire TE family: the family gets trapped. An important functional aspect is that production of piRNAs by piRNA loci requires maternal transmission of piRNAs both for repressing TE activity in the embryo germline and for stimulating piRNA production by the piRNA loci in the adult gonads [11,12]. Thus, in flies, a catalog of potentially dangerous sequences is transmitted from one generation to the other via cytoplasmic inheritance of piRNAs within the embryo. Worms also have a piRNA machinery but the strategy of transgenerational inheritance appears to be different. Indeed, two large clusters of piRNA loci produce thousands of different piRNAs that associate with the PIWI protein PRG-1 and target many sequences, as mismatches between piRNAs and their targets are tolerated $[13,14]$. When a sequence is targeted by piRNAs, the signal is amplified by an RNA-dependent RNA Polymerase (RdRP) and further relayed to a small interfering RNA pathway based on double strand RNA slicing performed by WAGO proteins [14-18]. Once this silencing is established, the piRNA machinery is no longer necessary and the information can be maintained in further generations solely by the WAGO protein pathway. The piRNA machinery has thus a crucial role for the establishment of silencing but not for its maintenance. To avoid too extensive, unspecific attack of transcripts by piRNAs, an active pathway exists, linked to production of small RNAs associated with a protein called CSR-1, which counteracts the effect of the piRNAs and consequently licenses transcripts for expression [16,19]. This CSR-1-associated RNAs are also transmitted from one generation to the other and allow transgenerational information of transcripts that have to be protected from degradation by piRNAs loaded onto PRG-1 [19]. 
It appears that in fly, one generation epigenetically transmits to the next a list of sequences to be repressed, whereas worms transmit information about both, correct repression or expression [20]. In both species, these systems were investigated using transgenes, and transgenic loci can exist at a given locus in opposite epigenetic states. In fly, clusters of transgenes can be stably maintained over generations, in either a quiescent or active state for production of abundant amounts of transgenehomologous piRNAs that establish trans-silencing of homologous transgene in the germline $[21,22]$. In worm, the same transgene can exist in an active or repressed state for their expression depending on the line [16]. The key point is that in both species, epigenetic conversion processes can occur in the germline between repressed and active loci, which can further be transmitted to the next generations in the absence of the converting locus. It results in a paramutation likephenomenon, resembling this classical epigenetic conversion as observed in maize [1,2,23,24]. This review will describe properties of these epigenetic conversion processes and highlight the parallels and differences between the two models.

\section{2- Drosophila paramutation-like conversion}

The piRNA pathway was discovered in fly in 2006-2007 [3,4,25] and functionally validated with the use of genomic sites capable of transposon repression [11]. Indeed, two TEs invaded the genome of natural $D$. melanogaster populations in the $20^{\text {th }}$ century, the $P$ element (a DNA transposon) [26] and the I factor (a LINE retroelement) [27]. For the two TEs, it was shown that the cross of females devoid of TE copies (from lines collected before the invasion) with males carrying numerous copies of the TE (from lines collected after the invasion) produces progeny showing a syndrome of genetic abnormalities in the germline called hybrid dysgenesis (high mutation rate, chromosomal breakages, thermo-sensitive sterility) [28,29]. Cytoplasmic inheritance was shown to play a key role as reciprocal crosses (TE-bearing females x TE-devoid males) produced progeny without dysgenesis [29-32]. Thus cytoplasm from females devoid of $P$ or $I$ elements was therefore missing “something” [33-35], later shown to be $P$ - or $I$-homologous piRNAs $[11,36]$. For the $P$ element, a master locus for repressing hybrid dysgenesis was identified at the telomere of the $X$ chromosome [37-40], within subtelomeric heterochromatin called Telomeric Associated Sequences (TAS) [41]. Such telomeric heterochromatin was shown to produce abundant ovarian piRNAs [3]. In addition, $P$ copies inserted in TAS were also shown to produce abundant ovarian piRNAs [11,21,42]. A transgenic system based on $P$-derived sequences located in TAS was developed and used to study the phenotypic and genetic properties of piRNA-mediated repression in the germline. 
In this system, called Trans-Silencing Effect (TSE), a $P$-transgene located in TAS can repress a homologous transgene in the female germline [12,43,44]. Inheritance of this repressive capacity shows both a maternal effect and a partial persistence of the maternal effect over up to 6 generations [12]. TSE studies and a mutant approach allowed to confirm that all the piRNA genes tested were necessary for the trans-silencing capacities of telomeric $P$ insertions $[12,43,45]$. Finally, the use of TSE also allowed to discover that some other structures in the genome can establish piRNAmediated repression [22]. Such studies revealed puzzling situations as the two lines $T-1$ and $B X 2$ carrying a similar cluster of P-lacZ-white transgenes exhibit different properties. Indeed the $T-1$ induces a strong TSE whereas $B X 2$ showed no repression capacity [22]. These $P$-lacZ-white clusters show stochastic on-off repression of the white marker in the eye (called variegation). This variegation results from Repeat Induced Gene Silencing (RIGS) [46,47] and is associated with the local binding of Heterochromatin Protein 1 (HP1) at the cluster, as tested by immuno-staining of salivary gland polytene chromosomes [48]. $T-1$ has the same cluster than $B X 2$ but carries chromosomal inversions and translocations induced by X-ray treatment. The trans-silencing capacities of $T-1$ and $B X 2$ appeared stable over more than one decade for the two lines. This allowed investigating possible factors that could activate piRNA production by the quiescent $B X 2$ locus.

It was then tested if maternal inheritance of P-lacZ-white homologous piRNAs could de novo activate the production of piRNAs from an initially quiescent $B X 2$ locus [21,49]. $T-1$ heterozygous females were crossed with $B X 2$ males. Female progeny having inherited the $B X 2$ locus and a $T-1$ cytoplasm, but not the $T$-1 locus, produced ovarian P-lacZ-white piRNAs and induced a complete trans-silencing (Figure 1). The phenomenon showed complete penetrance in each experiment. We called $B X 2^{*}$ these paramutated $B X 2$ females by contrast to the initial "BX2 naïve" (nonparamutated) flies producing no P-lacZ-white piRNAs. $B X 2 *$ lines were established and further studied for their capacity to induce TSE and produce piRNAs. Again all the tested lines (more than 20) exhibited stable repression. One line was investigated for very long terms: it still induced complete TSE after more than 120 generations, and ovarian $P$-lacZ-white piRNA production was confirmed at $\mathrm{G}_{42}$ and $\mathrm{G}_{83}$. It was also tested if the $B X 2 *$ line is paramutagenic by crossing $B X 2 *$ females at $\mathrm{G}_{42}$ with $B X 2$ naive males. The progeny (called $B X 2^{* *}$ ) having inherited a paternal - but not maternal - BX2 locus showed complete TSE capacity and produced abundant P-lacZ-white piRNAs in ovaries (Figure 1). $B X 2^{*}$ females are thus fully paramutagenic. Similarly, $B X 2^{* *}$ females are also completely paramutagenic. Therefore, epigenetic conversion occurs between $B X 2 *$ 
and $B X 2$ naive loci; the change is heritable, and the process can be repeated. This epigenetic conversion presents the properties of a paramutation which is mediated via cytoplasmic inheritance: this excludes any role of pairing between the loci in the paramutation process. As control, $T-1$ males were crossed to $B X 2$ females, and the progeny having inherited the $B X 2$ locus did not show any trans-silencing capacities. Resulting flies carrying the $B X 2$ loci failed to show silencing capacities in further generations (our lab, unpublished). Further, the paramutation process can be erased by crossing $B X 2 *$ males with females that do not carry any $P$-lacZ-white transgene. This clearance is stable for over than 50 generations (our lab, unpublished). To exclude the hypothesis that an autoreproductive cytoplasmic component would be sufficient to induce paramutation, we also have performed a "passage à vide” (empty step) experiment [49]: $B X 2 *$ females were crossed with males devoid of P-lacZ-white transgene and $G_{1}$ females which did not inherit the $B X 2 *$ cluster were crossed with $B X 2$ naïve males in order to test if their cytoplasm can induce paramutation. $G_{2}$ females, whose grand-mother produced P-lacZ-white piRNAs, but whose mother were unable to maintain such production due to the lack of P-lacZ-white transgene, showed no repression capacities confirming that this paramutation does not result from an autoreplicative cytoplasmic component.

$B X 2 *$ females produce not only abundant P-lacZ-white 23-28nt piRNAs but also abundant 21nt RNAs [21]. We have tested the effect of a homozygous mutation of Dicer-2, involved in the siRNA pathway on $B X 2 *$ properties. The trans-silencing capacities were unaffected by a Dicer-2 loss of function, even after 50 generations in a homozygous mutant context. We have also tested the effect of a heteroallelic mutant context for the PIWI family gene aubergine on $B X 2 *$ females: silencing capacities were completely abolished in such mutant females, showing that paramutation requires piRNAs but not siRNAs.

What is the molecular mechanism of paramutation linked to piRNAs in Drosophila? It can be assumed that maternally transmitted piRNAs are incorporated in Primordial Germ Cells (PGCs) in embryos and will potentiate piRNA production by homologous loci $[49,50]$. One possibility is that piRNAs associated with the Piwi protein reach the PGC nuclei and interact with the nascent transcripts on the piRNA locus to promote the binding of the Rhino protein, a member of the Heterochromatin Protein 1 (HP1) subfamily of chromo box proteins [51], which was shown to have a causal role on piRNA production [6]. Indeed, PIWI loaded with a piRNA has the capacity to enter the nucleus. Such hypothesis is supported by chromatin immunoprecipitation (ChIP) experiments performed on $B X 2 *$ vs $B X 2$ naive ovaries showing an enrichment of posttranslational methylation of 
histone H3 (H3K9me3) on the cluster when the locus is paramutated [50]. H3K9me3 residues can indeed be targeted by the chromodomain of Rhino, resulting in Rhino-associated protein complex stabilization on the cluster. Alternatively, piRNAs loaded on the PIWI proteins Aubergine and Ago3 can also be transmitted to PGC in embryos where they could stimulate the amplification step of the piRNA biogenesis, which occurs in the nuage, a peri-nuclear diffuse structure surrounding the germline cell nucleus and enriched in PIWI proteins. In both models, paramutation would be induced by a diffusible cytoplasmic product, the piRNAs.

\section{3- Worm paramutation-like conversions}

Transgenesis in worms showed early on that not all single copy transgenes were expressed in the germline and that failure of expression appeared to be linked to the length of foreign DNA present in the transgene construct. Repression of the transgene occurred at both the post-transcriptional and transcriptional levels and is associated with H3K9me3 enrichment present on the silenced transgene [16]. The cross of lines carrying expressed copies with lines carrying repressed ones surprisingly showed that, in a number of cases, the repressed state dominates in the progeny (Figure 2). For example, the cross of hermaphrodites carrying a gfp::csr-1 repressed transgene with males carrying an expressed gfp::csr-1 transgene produces progeny in which both transgenes are repressed in the germline (Figure 2-A) [16]. The analysis of succeeding generations further revealed that the repressed state of both transgenes is maintained. The same dominance and conversion process was observed between repressed and active gfp::csr-1 transgenes located on different chromosomes (Figure 2-B). Furthermore, the newly repressed transgene conserved its properties over generations, in absence of the initial silencer transgene. Such conversion phenomenon can be mediated by passage of the silenced copy through both parents but it is less efficient via male transmission [16]. In these experiments, the initial repressed transgene behaved as a paramutagenic locus and the newly repressed transgene, appearing stable for at least 10 generations in absence of the inducer locus, behaved as a paramutated locus. This silencing conversion was termed RNA-induced epigenetic Silencing (RNAe) [15,16]. The repressed loci were termed gfp::csr-1(RNAe) and the active loci $g f p:: c s r-1(+)$. The capacity of the paramutated locus to be paramutagenic was not tested, but it would probably work since $g f p$-transgene silencing mediated by $g f p$ dsRNA in presence of a transgene sensitive to RNAe is stable over generations [16]. Like in Drosophila, pairing between the two interacting loci during RNAe conversion is not likely to be necessary as conversion can occur when the transgenes are located on different chromosomes. This type of conversion was 
observed with different transgene structures (carrying $c d k-1$ instead of $c s r-1$ with the $g p f$ sequence). The presence of an RNAe transgene in the genome does not appear to induce co-suppression of homologous endogenous genes, as tested by western-blot [16].

Strikingly, not all transgenes undergo RNAe. Indeed, the gfp::wrm-1 and oma-1::gfp not only are resistant to the RNAe transgene-mediated repression but are able to switch some of them into an active state ([16], Figure 2 C and D). The activation process, called RNA-induced epigenetic gene activation (RNAa), is only progressive and not as fast as RNAe since several generations of coexistence of the two transgenes in the same genome are necessary to induce full activation of the repressed transgene or to confer it stability in next generations, after out-cross with the wild-type removing the initial active transgene [19]. For example, the combination of oma-1::gfp (RNAa) with $g f p-c d k-1(R N A e)$ results in activation of the latter transgene. Segregation after only one generation of contact between the two transgenes results in immediate reversion of the $g f p$-cdk-1 transgene to the repressed state. Segregation after ten generations of contact between the two transgenes provides stability for one generation to $g f p$ - $c d k-1$, and 30 generations of contact provide a stability to the activated transgene for 8-10 generations after segregation away from the activating locus (Figure $2 \mathrm{C}$ and D). Some transgenes, however are resistant to RNAa since a gfp::csr-1 (RNAe) transgene is insensitive to the presence of the oma-1::gfp transgene: in that case, the two transgenes keep their own properties in a double transgenic line and in succeeding generations after cross to wild-type $[16,19]$.

More recently, a case of “cascade” RNAe paramutation was described in Caenorhabditis elegans using a different combination of transgenes [52]. The first step involves on the one hand a "piRNA sensor" transgene carrying a gfp gene and a piRNA-targeted locus (21UR-1), and on the other hand an "operon" transgene which expresses mCherry and $g f p$ mRNAs from a single primary transcript (Figure 3). mCherry is located upstream of $g f p$ within this operon. The strain carrying the piRNA sensor transgene exhibits no $g f p$ expression in the germline due to the targeting of the 21UR-1 sequence by piRNAs [14,17]. In contrast, the strain carrying the operon transgene shows strong $g f p$ and $m C h e r r y$ expression [52]. Combining the piRNA sensor transgene with the operon transgene by crossing produces progeny in which the operon transgene is repressed for both $\mathrm{gfp}$ and $m$ Cherry expression in the germline. Therefore, the operon transgene is trans-silenced by the piRNA sensor transgene, and silencing propagates from the $g f p$ sequence to the mCherry sequence in the operon. Out-crossing this progeny with worms devoid of any transgene generates lines carrying only the operon transgene that remains completely repressed over at least 12 generations ("operon (OFF)" 
lines). In a second step, crossing the operon (OFF) worms with worms carrying an active mcherry::H2A transgene, but no gfp sequence, produces progeny in which mCherry expression of both the operon and mcherry::H2A transgenes is completely repressed. Further, removal of the operon silencer transgene by out-crossing results in lines carrying the targeted mcherry::H2A transgene alone, and this remains repressed at least for 10 generations. Thus, the operon (OFF) transgene, which was paramutated by the piRNA sensor transgene, is stably repressed over generations and appears strongly paramutagenic since it can transfer inheritable silencing capacities to the mcherry::H2A transgene. Finally, this repressed mcherry::H2A transgene, which is a "second-order" paramutated locus, was also shown to be able to trans-inactivate other transgenes carrying the mcherry sequence. This indicates that the epigenetic conversion process can be recurrent. The conversion processes described here exhibit the properties of a bona fide paramutation. In these experiments, it was shown that paramutation can be performed by both hermaphrodite and paternal transmission of the paramutagenic locus but the effect appears stronger via hermaphrodite inheritance (Nic Lehrbach and Eric Miska, personal communication). Further, crossing hermaphrodites carrying an operon (OFF) transgene at the heterozygous state with males carrying a mcherry::H2A transgene generated individuals which did not inherit the operon (OFF) transgene but carried a repressed mcherry::H2A transgene with properties stably transmitted over generations [52]. This indicates that, like in fly, paramutation can be mediated by cytoplasmic components without inheritance of the paramutagenic locus itself, excluding again of the need for pairing between the loci in the process. Importantly, this combination of structurally different transgenes shows that silencing propagation within a transgene, from the targeted transgene part ( $g f p$ ) to the distal sequence (mCherry), is accompanied by production of paramutagenic signal from the distal sequence. Indeed, operon (OFF) worms produce siRNAs homologous to the mCherry sequence which are called tertiary siRNAs [52], by contrast to siRNAs produced by sequences located near the piRNA target site which are called secondary siRNAs [14]. These tertiary siRNAs can transfer heritable silencing capacities in trans and are thus paramutagenic.

Altogether, the different studies show that complete paramutation exists in worms and transgenes can occur in three different epigenetic states [15-17,19,52]: RNAe and RNAa transgenes and neutral transgenes that are expressed, but can be sensitive to the presence of RNAe transgenes [16,19]. However, these neutral alleles by themselves do not have the capacity to modify the expression of other homologous transgenes present in the same genome. What can be the mechanism of RNAe- and RNAa-linked epigenetic conversions? It appears that a complex balance 
of antagonistic systems determines the properties of a transgene and its capacity to affect expression of other homologous transgenes in trans. The establishment of the RNAe requires the PIWI protein PRG-1, and its maintenance depends on HPL-2, a HP1 ortholog, and several putative histone methyltransferases [15,17]. In addition, maintenance of the RNAe state requires proteins involved in the WAGO-mediated RNAi pathway such as RED3, MUT7, WAGO-1 or WAGO-9 [16]. The establishment of RNAa by contrast requires the Argonaute protein CSR-1 [19]. A dose effect is observed for CSR-1 since heterozygous individuals show impaired RNAa. CSR-1 engages siRNAs homologous to most of the germline-expressed genes [53,54] but CSR-1-interacting small RNAs (22G-RNAs) do not appear to induce silencing [53]. What could be the roles of these antagonistic systems at the genome level? Worms appear to have an opposite strategy to flies. In flies, the piRNA loci represent a repertoire of sequences that have to be repressed and are mostly homologous to mobile elements [3]. Therefore, piRNAs mainly target mobile sequences although they can also target some gene transcripts containing TE fragments [55]. By contrast, in worms, the two piRNA loci located on chromosome 4 produce around 15,000 different piRNAs, which can target transcripts despite incomplete homology (up to 4nt out of 21nt) [13,14,18,54]. Consequently, a very high number of gene transcripts can potentially be targeted by piRNAs, and a protecting system is thus required to ensure gene expression. This system appears to be mediated by the 22G RNAs loaded on the CSR-1 protein, which might protect homologous transcript from the effect of 21-U PRG-1 RNAs. Antagonist 22G CSR-1 RNAs and 21-U PRG-1 RNAs would be therefore able to exert opposite effects on their common homologous transcript targets. If the effect of PRG-1 dominates, the transcript binds an RdRP that produces double-strand RNAs further targeted by the WAGO-RNAi machinery, leading to production of 22-G WAGO RNAs. These WAGO-RNAs can then induce both PTGS of homologous transcripts and TGS of homologous loci $[54,56]$. If the effect of CSR-1 dominates, there is no double-strand RNA production by RdRP and the siRNA machinery is not activated. So, the piRNA machinery is required for the silencing establishment but not for its maintenance over generations, which relies on siRNA activation $[15,17,57]$. Cytoplasmic trans-generational transmission includes information carried in both WAGO interacting RNAs as a memory of silenced targets and CSR-1 interacting RNAs as a memory of targets licensed for expression. The PRG-1 system is involved in the foreign DNA detection step (which was not previously licensed) and the WAGO machinery is required for amplification and maintenance of the information and repression itself. 
It appears that maintenance of the properties of epigenetically repressed RNAe or activated RNAa transgenes, each alone in a genome, involves not only cytoplasmic RNA molecules associated with WAGO (silencing) or CSR-1 (activation), but also chromatin marks on the locus deposited via either a WAGO RNAs or potentially a CSR-1 RNA-dependent mechanism for silencing or activation, respectively $[16,19,52,58]$. Two opposite loops between small RNAs and chromatin could exist for RNAe and RNAa transgenes, and the maintenance of the loop from one generation to the other would be established by cytoplasmic inheritance of WAGO RNAs for RNAe and CSR1 RNAs for RNAa. When the two types of transgenes are present in the same genome, different small RNAs might compete both at the cytoplasmic and nuclear level. In the cytoplasm, a given transcript could be confronted with 21-U PRG-1 RNAs (and 22G WAGO RNAs) in one hand and with antagonist CSR-1 RNAs on the other hand. For RNAe conversion process, a strong RNAe transgene would generate a high quantity of 22G WAGO RNAs, which could potentially counteract a previously established presence of CSR-1 RNAs. By contrast, for an RNAa conversion process, a strong RNAa allele would generate a high quantity of CSR-1 RNAs that could counteract the effects of previously established RNAe alleles. In the nucleus, the 22G RNAs brought by RNAe or RNAa alleles could displace chromatin tags on the antagonist transgenes. For example, 22G WAGO RNAs could potentially trigger removal of CSR-1 from the chromatin of an RNAa allele and thus break a positive loop in which CSR-1 present on the locus favors the production of 22G CSR-1 RNAs. Reciprocally, 22G CSR-1 RNAs could displace chromatin tags on RNAe alleles similarly involved in a positive loop. In any case, this system implies that for a gene to be expressed, it is necessary that it produces sufficient quantity of RNA for first, establishing a population of CSR-1 RNAs to counteract silencing machinery and second, for being translated. Neutral transgenes alone in a genome would escape RNAe silencing and could be thus expressed. However, in the presence of strong RNAe, neutral alleles would be switched towards RNAe at the cytoplasmic and /or chromatin levels. When the RNAe-inducing allele is removed by crosses to wild-type, repression loops now associated to the repressed targeted transgenes could be maintained by themselves over at least some generations. It appears that the RNAe-induced conversion is relatively stable. This is probably due to the strength of the 22G-RNAs WAGO production based on RdRP activity. Finally, “adoption” of foreign sequences can be observed linked to cis-spreading of CSR-1 on a transgene which contains adjacent endogenous and foreign sequences [19]. 


\section{Conclusion}

Flies and worms can undergo epigenetic conversions showing paramutation-like properties. In fly, the conversion is unidirectional, corresponding to the transfer of a homology-dependent silencing capacity from one locus to the next, which reflects the capacity to produce piRNAs [21,49]. The conversion acts on the piRNA producing locus itself. It includes a change on the locus since the chromatin of the new piRNA-producing locus is associated with enriched H3K9 histone methylation marks [50]. In worm, the conversion can occur in two directions, probably reflecting the competition of two antagonist regulation loops whose strength can vary. Apparently, it does not involve the piRNA loci themselves but rather their targeted sequences. It appears, however, that the change toward the repressed state is more stable over generations than the opposite change. This RNAe conversion presents the stability of a true paramutation and can be recurrent. By contrast, the RNAa process appears not only less stable but the activated alleles are also not able to trigger further RNAa conversion [19]. So RNAa conversion does not exhibit all the properties of a complete paramutation. In fly, conversion is mediated only via maternal cytoplasmic transmission, in contrast to worms in which RNAe conversion can be mediated by transmission from both parents, possibly because of different proportions of paternal cytoplasmic transmission in the two species. A key point for future work will be to determine if some antagonistic forces may also exist in fly to counteract the action of piRNAs. Furthermore, in both species it will be essential to characterize the developmental events involved in the transgenerational transmission of these conversion processes.

Acknowledgements: SR thanks Doug Dorer, Steve Henikoff, and the Bloomington Stock Center for providing stocks, and flybase.org for providing databases. SR thanks Nic Lehrbach and Eric Miska for personal communication. SR thanks Catherine Hermant for Figure 1, Safia Deddouche Grass, Laure Teysset, Antoine Boivin, Amna Asif-Laidin, Neel Randsholt and an anonymous reader for their help in preparing the manuscript. SR thanks all the authors of the de Vanssay et al. 2012 paper for their investigation of fly paramutation and for helpful discussions. SR especially thanks Valérie Delmarre for technical help and unpublished results. This work was supported by fellowships from the Ministère de l'Enseignement Supérieur et de la Recherche (ED515), by grants from the Association de la Recherche contre le Cancer (Fondation ARC, SFI 20121205921, PJA 20131200470), from the Fondation pour la Recherche Médicale (FRM DEP20131128532) and from the Association Nationale de la Recherche (ANR, project "plastisipi"). 


\section{References.}

1. Chandler VL (2007) Paramutation: from maize to mice. Cell 128: 641-645.

2. Brink RA (1956) A Genetic Change Associated with the R Locus in Maize Which Is Directed and Potentially Reversible. Genetics 41: 872-889.

3. Brennecke J, Aravin AA, Stark A, Dus M, Kellis M, et al. (2007) Discrete small RNA-generating loci as master regulators of transposon activity in Drosophila. Cell 128: 1089-1103.

4. Gunawardane LS, Saito K, Nishida KM, Miyoshi K, Kawamura Y, et al. (2007) A slicer-mediated mechanism for repeat-associated siRNA $5^{\prime}$ end formation in Drosophila. Science 315: 1587-1590.

5. Mohn F, Sienski G, Handler D, Brennecke J (2014) The rhino-deadlock-cutoff complex licenses noncanonical transcription of dual-strand piRNA clusters in Drosophila. Cell 157: 13641379.

6. Zhang Z, Wang J, Schultz N, Zhang F, Parhad SS, et al. (2014) The HP1 homolog rhino anchors a nuclear complex that suppresses piRNA precursor splicing. Cell 157: 1353-1363.

7. Sato K, Siomi MC (2013) Piwi-interacting RNAs: biological functions and biogenesis. Essays Biochem 54: 39-52.

8. Weick EM, Miska EA (2014) piRNAs: from biogenesis to function. Development 141: 3458-3471.

9. Le Thomas A, Rogers AK, Webster A, Marinov GK, Liao SE, et al. (2013) Piwi induces piRNAguided transcriptional silencing and establishment of a repressive chromatin state. Genes Dev 27: 390-399.

10. Sienski G, Donertas D, Brennecke J (2012) Transcriptional silencing of transposons by Piwi and maelstrom and its impact on chromatin state and gene expression. Cell 151: 964-980.

11. Brennecke J, Malone CD, Aravin AA, Sachidanandam R, Stark A, et al. (2008) An epigenetic role for maternally inherited piRNAs in transposon silencing. Science 322: 1387-1392.

12. Josse T, Teysset L, Todeschini AL, Sidor CM, Anxolabehere D, et al. (2007) Telomeric transsilencing: an epigenetic repression combining RNA silencing and heterochromatin formation. PLoS Genet 3: 1633-1643.

13. Batista PJ, Ruby JG, Claycomb JM, Chiang R, Fahlgren N, et al. (2008) PRG-1 and 21U-RNAs interact to form the piRNA complex required for fertility in C. elegans. Mol Cell 31: 67-78.

14. Bagijn MP, Goldstein LD, Sapetschnig A, Weick EM, Bouasker S, et al. (2012) Function, targets, and evolution of Caenorhabditis elegans piRNAs. Science 337: 574-578.

15. Luteijn MJ, van Bergeijk P, Kaaij LJ, Almeida MV, Roovers EF, et al. (2012) Extremely stable Piwi-induced gene silencing in Caenorhabditis elegans. EMBO J 31: 3422-3430.

16. Shirayama M, Seth M, Lee HC, Gu W, Ishidate T, et al. (2012) piRNAs initiate an epigenetic memory of nonself RNA in the $C$. elegans germline. Cell 150: 65-77.

17. Ashe A, Sapetschnig A, Weick EM, Mitchell J, Bagijn MP, et al. (2012) piRNAs can trigger a multigenerational epigenetic memory in the germline of $C$. elegans. Cell 150: 88-99.

18. Das PP, Bagijn MP, Goldstein LD, Woolford JR, Lehrbach NJ, et al. (2008) Piwi and piRNAs act upstream of an endogenous siRNA pathway to suppress Tc3 transposon mobility in the Caenorhabditis elegans germline. Mol Cell 31: 79-90.

19. Seth M, Shirayama M, Gu W, Ishidate T, Conte D, Jr., et al. (2013) The C. elegans CSR-1 argonaute pathway counteracts epigenetic silencing to promote germline gene expression. Dev Cell 27: 656-663.

20. Conine CC, Moresco JJ, Gu W, Shirayama M, Conte D, Jr., et al. (2013) Argonautes promote male fertility and provide a paternal memory of germline gene expression in $C$. elegans. Cell 155: 1532-1544.

21. de Vanssay A, Bouge A-L, Boivin A, Hermant C, Teysset L, et al. (2012) Paramutation in Drosophila linked to emergence of a piRNA-producing locus. Nature 490: 112-115.

22. Ronsseray S, Boivin A, Anxolabehere D (2001) P-Element repression in Drosophila melanogaster by variegating clusters of P-lacZ-white transgenes. Genetics 159: 1631-1642.

23. Coe EH (1959) A regular and continuing conversion-type phenomenon at the B locus in maize. Proceedings of the National Academy of Sciences of the United States of America 45: 828832. 
24. Brink RA (1958) Paramutation at the R locus in maize. Cold Spring Harb Symp Quant Biol 23: 379-391.

25. Vagin VV, Sigova A, Li C, Seitz H, Gvozdev V, et al. (2006) A distinct small RNA pathway silences selfish genetic elements in the germline. Science 313: 320-324.

26. Engels WR (1989) P elements in Drosophila. Berg DE, Howe, M. M., editor. American Society for Microbiology, Washington, D.C. 437-484 p.

27. Finnegan DJ (1989) The I factor and I-R Hybrid Dysgenesis in Drosophila melanogaster. Mobile DNA Chp. 18: 503-518.

28. Kidwell MG, Kidwell JF, Sved JA (1977) Hybrid dysgenesis in Drosophila melanogaster: a syndrome of aberrant traits including mutation, sterility, and male recombination. Genetics 86: 813-833.

29. Picard G, Bregliano JC, Bucheton A, Lavige JM, Pelisson A, et al. (1978) Non-mendelian female sterility and hybrid dysgenesis in Drosophila melanogaster. Genet Res 32: 275-287.

30. Engels W R (1979) Hybrid dysgenesis in Drosophila melanogaster: rules of inheritance of female sterility. Genet Research 33: 219-236.

31. Engels WR (1979) Extrachromosomal control of mutability in Drosophila melanogaster. Proc Natl Acad Sci U S A 76: 4011-4015.

32. Picard G (1978) Non mendelian female sterility in Drosophila melanogaster: sterility in the daughter progeny of SF and RSF females. . Biol Cell 31: 235-244.

33. Ronsseray S, Lemaitre B, Coen D (1993) Maternal inheritance of P cytotype in Drosophila melanogaster: a "pre-P cytotype" is strictly extra-chromosomally transmitted. Mol Gen Genet 241: 115-123.

34. Niemi JB, Raymond JD, Patrek R, Simmons MJ (2004) Establishment and maintenance of the P cytotype associated with telomeric P elements in Drosophila melanogaster. Genetics 166: 255-264.

35. Bucheton A, Bregliano JC (1982) The I-R system of hybrid dysgenesis in Drosophila melanogaster: heredity of the reactive condition. Biol Cell 46: 123-132.

36. Grentzinger T, Armenise C, Brun C, Mugat B, Serrano V, et al. (2012) piRNA-mediated transgenerational inheritance of an acquired trait. Genome Res 22: 1877-1888.

37. Ronsseray S, Lehmann M, Anxolabehere D (1991) The maternally inherited regulation of $P$ elements in Drosophila melanogaster can be elicited by two P copies at cytological site 1A on the X chromosome. Genetics 129: 501-512.

38. Ronsseray S, Lehmann M, Nouaud D, Anxolabehere D (1996) The regulatory properties of autonomous subtelomeric $\mathrm{P}$ elements are sensitive to a Suppressor of variegation in Drosophila melanogaster. Genetics 143: 1663-1674.

39. Stuart JR, Haley KJ, Swedzinski D, Lockner S, Kocian PE, et al. (2002) Telomeric P elements associated with cytotype regulation of the $\mathrm{P}$ transposon family in Drosophila melanogaster. Genetics 162: 1641-1654.

40. Marin L, Lehmann M, Nouaud D, Izaabel H, Anxolabehere D, et al. (2000) P-Element repression in Drosophila melanogaster by a naturally occurring defective telomeric P copy. Genetics 155: 1841-1854.

41. Karpen GH, Spradling AC (1992) Analysis of subtelomeric heterochromatin in the Drosophila minichromosome Dp1187 by single P element insertional mutagenesis. Genetics 132: 737753.

42. Muerdter F, Olovnikov I, Molaro A, Rozhkov NV, Czech B, et al. (2011) Production of artificial piRNAs in flies and mice. RNA.

43. Josse T, Maurel-Zaffran C, de Vanssay A, Teysset L, Todeschini AL, et al. (2008) Telomeric trans-silencing in Drosophila melanogaster: tissue specificity, development and functional interactions between non-homologous telomeres. PLoS ONE 3: e3249.

44. Roche SE, Rio DC (1998) Trans-silencing by $P$ elements inserted in subtelomeric heterochromatin involves the Drosophila Polycomb group gene, Enhancer of zeste. Genetics 149: 1839-1855. 
45. Todeschini AL, Teysset L, Delmarre V, Ronsseray S (2010) The Epigenetic Trans-Silencing Effect in Drosophila Involves Maternally-Transmitted Small RNAs Whose Production Depends on the piRNA Pathway and HP1. PLoS One 5: e11032.

46. Dorer DR, Henikoff S (1994) Expansions of transgene repeats cause heterochromatin formation and gene silencing in Drosophila. Cell 77: 993-1002.

47. Dorer DR, Henikoff S (1997) Transgene repeat arrays interact with distant heterochromatin and cause silencing in cis and trans. Genetics 147: 1181-1190.

48. Fanti L, Dorer DR, Berloco M, Henikoff S, Pimpinelli S (1998) Heterochromatin protein 1 binds transgene arrays. Chromosoma 107: 286-292.

49. de Vanssay A, Bouge AL, Boivin A, Hermant C, Teysset L, et al. (2013) piRNAs and epigenetic conversion in Drosophila. Fly (Austin) 7: 237-241.

50. Le Thomas A, Stuwe E, Li S, Du J, Marinov G, et al. (2014) Transgenerationally inherited piRNAs trigger piRNA biogenesis by changing the chromatin of piRNA clusters and inducing precursor processing. Genes Dev 28: 1667-1680.

51. Volpe AM, Horowitz H, Grafer CM, Jackson SM, Berg CA (2001) Drosophila rhino encodes a female-specific chromo-domain protein that affects chromosome structure and egg polarity. Genetics 159: 1117-1134.

52. Sapetschnig A, Sarkies P, Lehrbach NJ, Miska EA (2015) Tertiary siRNAs mediate paramutation in C. elegans. PLoS Genet 11: e1005078.

53. Claycomb JM, Batista PJ, Pang KM, Gu W, Vasale JJ, et al. (2009) The Argonaute CSR-1 and its 22G-RNA cofactors are required for holocentric chromosome segregation. Cell 139: 123134.

54. Gu W, Shirayama M, Conte D, Jr., Vasale J, Batista PJ, et al. (2009) Distinct argonautemediated 22G-RNA pathways direct genome surveillance in the $C$. elegans germline. Mol Cell 36: 231-244.

55. Rouget C, Papin C, Boureux A, Meunier AC, Franco B, et al. (2010) Maternal mRNA deadenylation and decay by the piRNA pathway in the early Drosophila embryo. Nature 467: 1128-1132.

56. Buckley BA, Burkhart KB, Gu SG, Spracklin G, Kershner A, et al. (2012) A nuclear Argonaute promotes multigenerational epigenetic inheritance and germline immortality. Nature 489: 447-451.

57. Lee HC, Gu W, Shirayama M, Youngman E, Conte D, Jr., et al. (2012) C. elegans piRNAs mediate the genome-wide surveillance of germline transcripts. Cell 150: 78-87.

58. Wedeles CJ, Wu MZ, Claycomb JM (2013) Protection of germline gene expression by the C. elegans Argonaute CSR-1. Dev Cell 27: 664-671. 


\section{Figure 1: Paramutation linked to piRNAs in fly.}

A tandemly repeated P-lacZ-white transgenic locus, called $T$-1, which produces abundant piRNAs in ovaries and induces complete silencing of a homologous transgenic target in the germline was used as a paramutagenic locus. A similar transgenic locus, called $B X 2$, located at the same genomic site, and stably devoid of piRNA production and trans-silencing capacities was used as paramutable locus. The mating scheme is shown with the number of generations indicated on the left. CyRoi and Cy are balancer chromosomes carrying distinct dominant phenotypic makers. Orange color indicates cytoplasm inheritance. Histograms show the length distributions (19-29 nt) of ovarian small RNAs matching P-lacZ-white. Positive (red) and negative (blue) bars correspond to sense and antisense reads, respectively. Trans-silencing capacities were measured by crossing tested females with males carrying a single $P$-lac $Z$ transgene expressed in the female germline and by counting the percentage of egg chambers showing target transgene repression $(n=$ total number of egg chambers counted). $\mathrm{G}_{1}$ females having paternally inherited the $B X 2$ locus and having maternally inherited a $T$ 1 cytoplasm, but not the $T-1$ locus itself were generated. Lines established from $\mathrm{G}_{1}$ individuals, called $B X 2^{*}$ (first order paramutation), were further analyzed. $B X 2^{*}$ females produce abundant transgene-homologous piRNAs and induce a complete trans-silencing in the germline as tested in $\mathrm{G}_{2}$ and $\mathrm{G}_{83}$. Finally, females from $B X 2 *$ lines (at $\mathrm{G}_{42}$ ) were crossed with males of a $B X 2$ line quiescent for piRNA production and trans-silencing. This generated $B X 2^{* *}$ females, having paternally inherited the $B X 2$ locus and maternally inherited a $B X 2^{*}$ cytoplasm - but not the locus -, which produce abundant ovarian piRNAs and induce strong silencing. $B X 2^{* *}$ lines (second order paramutation) stably maintain these properties over generations. This recurrent conversion process has been repeated five times "en cascade", generating $B X 2 *^{5}$ lines showing a complete transsilencing capacity [21]. Thus, this epigenetic conversion process presents functional properties of paramutation. This process does not require the transmission of the paramutagenic locus but requires cytoplasm inheritance. 


\section{Figure 2: Reciprocal epigenetic conversions in worms.}

Single copies of the $g f p:: c s r-1$ transgene can exhibit $g f p$ expression or repression depending on PRG-1-associated piRNAs targeting. At the same genomic location, the two states can exist and appear stable over several generations. RNAe (RNA-induced epigenetic silencing): (A) The cross of hermaphrodites worms carrying a gfp::csr-1(RNAe) repressed transgene (grey $g f p$ box on the transgene map) with males carrying an expressed $g f p:: c s r-1(+)$ transgene (green $g f p$ box), both located on chromosome 2 (LGII), results in a progeny in which the two transgenes are repressed in the germline. Propagation of the derived lines shows that this repressed state is stable over generations at least until $G_{10}$ (B) Similar silencing conversion can occur between transgenes located on different chromosomes. The cross of hermaphrodites carrying a gfp::csr-1(RNAe) repressed transgene located on chromosome 4 (LGIV) with males carrying an expressed gfp::csr$1(+)$ transgene located on chromosome 2 , also results in progeny in which the two transgenes are repressed. The repressed state of the transgene located on chromosome 2 is maintained in successive generations after the removal of the silencer transgene located on chromosome 4 by crosses. Such RNAe conversion can occur during inheritance from both parents. RNAa (RNAinduced epigenetic gene activation): (C) Some active transgenes appear to be resistant to the effect of RNAe transgenes and show opposite properties. The cross of hermaphrodites carrying an active oma1::gfp(RNAa) transgene (chromosome 4) with males carrying a repressed gfp::cdk-1(RNAe) transgene (chromosome 2) results in progeny showing gfp expression of both transgenes. If the two transgenes are separated after one generation by out-crossing to wild-type, the $g f p:: c d k$ - 1 transgene is immediately re-silenced [19]. Out-crossing after ten generations of double transgenic strains allows persistence of the $g f p:: c d k-1$ active state for one generation, but then the effect is lost later. (D) Propagation of the double transgenic strain for 30 generations allows maintaining of a $g f p:: c d k$ 1 fully active state for 8 generations after out-crossing, before transgene activation decreases. By contrast to RNAe, which can be stably established in one generation, RNAa needs a longer exposure and only results in a transitory active state. (Adapted from [16,19]). 


\section{Figure 3: Cascade of RNAe paramutations in worms.}

The piRNA sensor transgene is targeted by an endogenous piRNA (21UR-1) specific for the "21U" site and shows no gfp expression. This repression correlates with production of siRNAs by the $g f p$ sequence. Crossing worms carrying a piRNA sensor repressed transgene with worms carrying an operon expressed transgene generates individuals in which the operon transgene is repressed for both $g f p$ and $m$ Cherry expression in the germline. This repression correlates with cis-propagation of the capacity to produce siRNAs from the gfp until the mCherry sequence. This "operon (OFF)" transgene remains repressed in the absence of piRNA sensor transgene after outcrossing. Crossing worms carrying an operon (OFF) transgene with worms carrying a mcherry::H2A expressed transgene produces individuals in which both mCherry transgenes are repressed. As before, the mcherry::H2A transgene remains repressed in the absence of operon (OFF) transgene after outcrossing. Finally, crossing individuals carrying a repressed mcherry::H2A (OFF) transgene with individuals carrying a mcherry expressed transgene produces progeny in which both transgenes exhibit mcherry repression. The operon (OFF) transgene is thus paramutated by the piRNA sensor transgene (first order paramutation) and can paramutate a mcherry transgene (second order paramutation). (Adapted from [52]). 
Figures 1-3

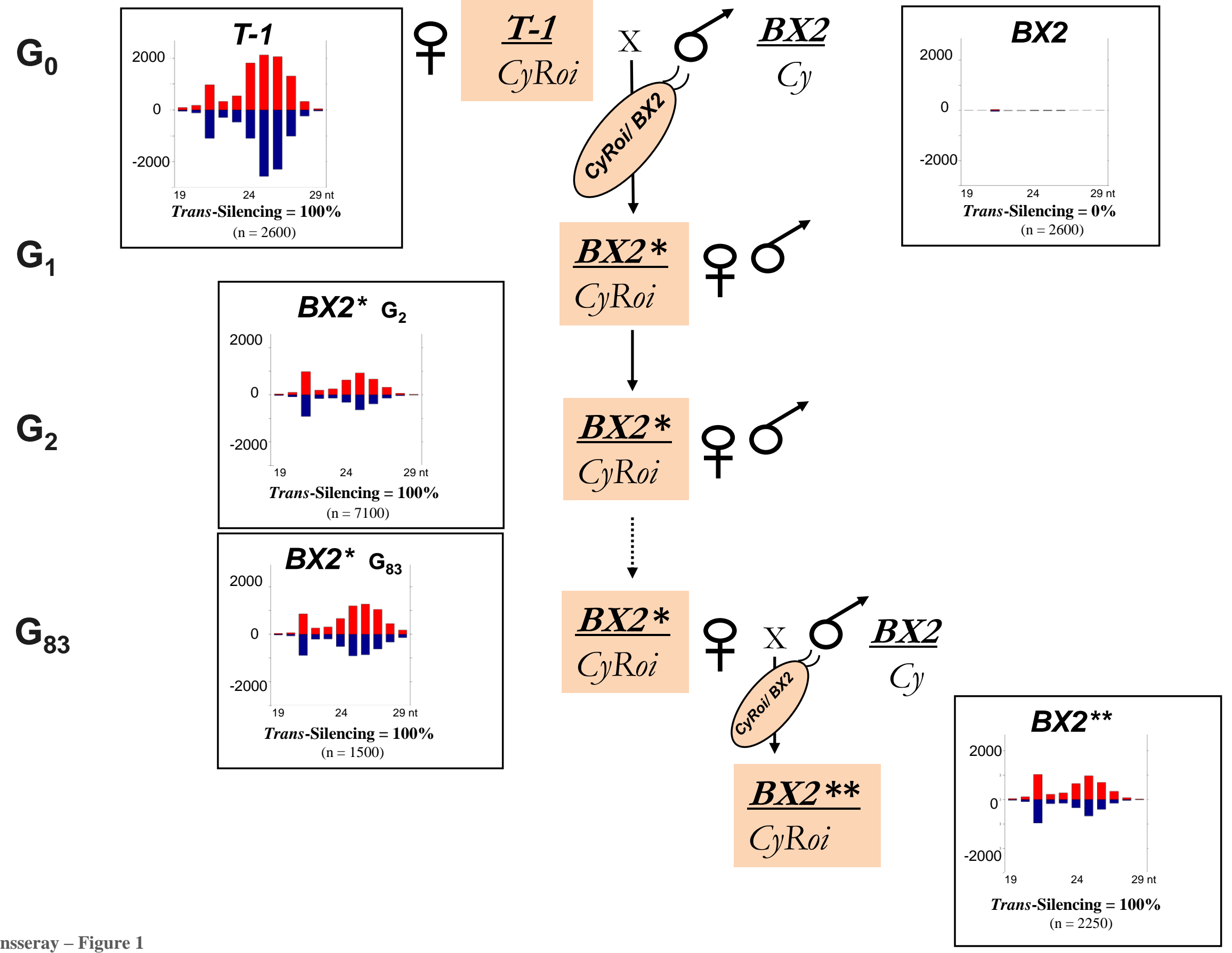




\section{Silencing conversion (RNAe)}

A

B
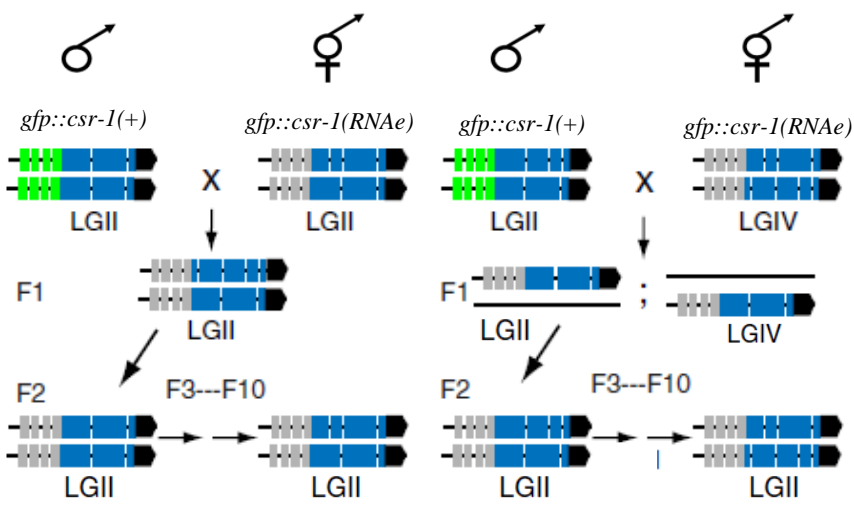

\section{Activating conversion (RNAa)}

C

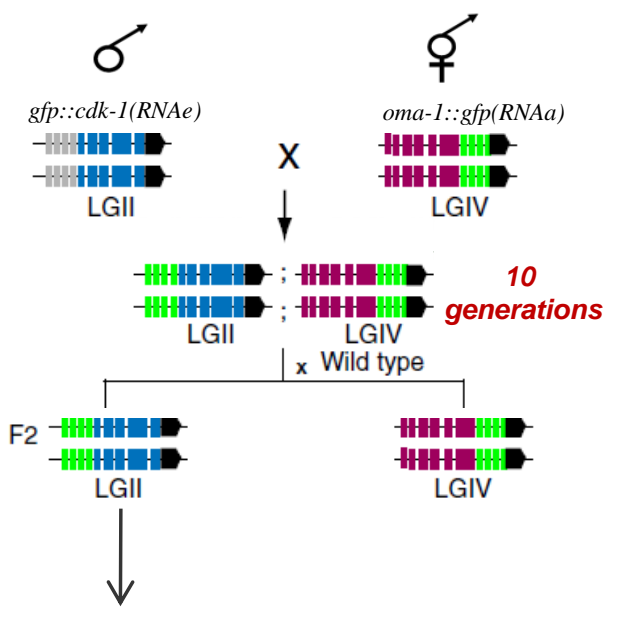

F3: $0 \%$ GFP+
D

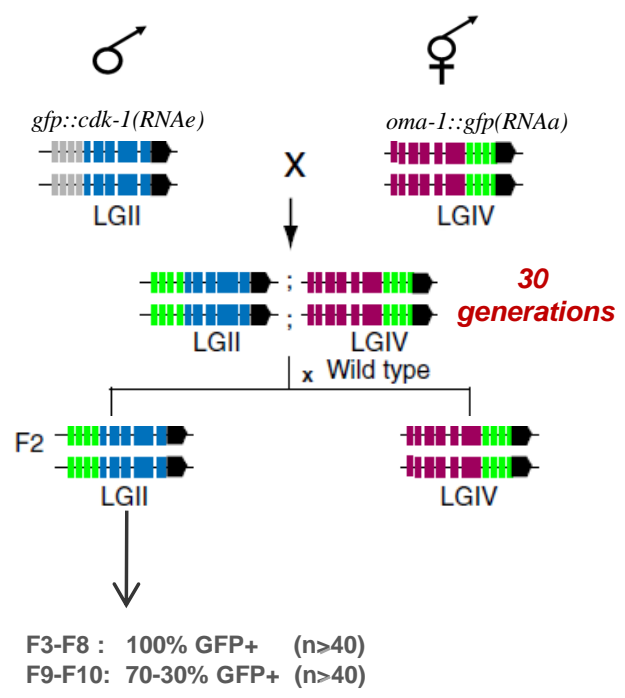


piRNA locus

piRNA sensor

$$
\text { operon (OFF) }
$$

\section{mcherry::H2A (OFF)}

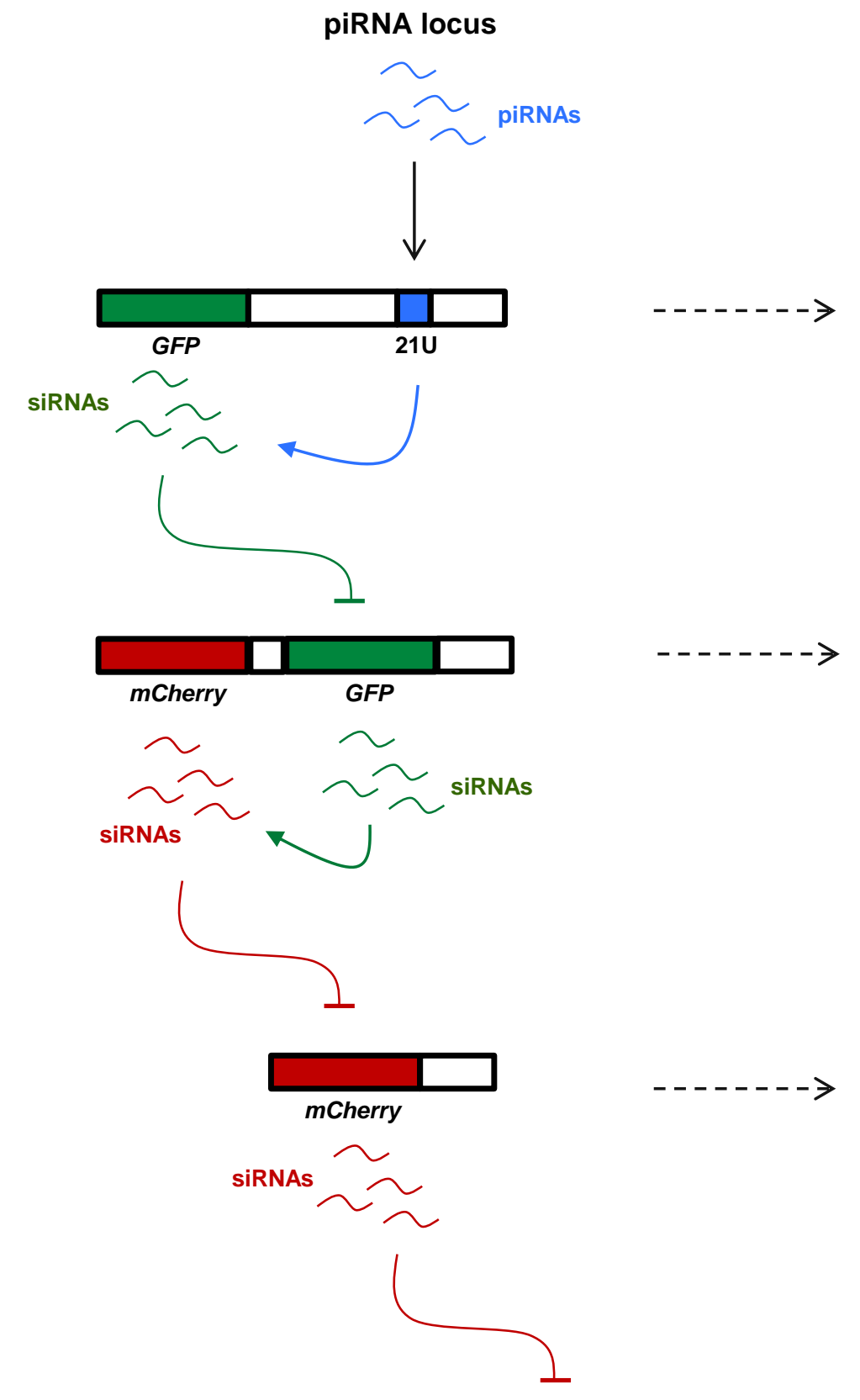

trans-silencing of another mcherry transgene
GFP expression stably repressed over generations

mCherry expression stably repressed over generations after removal of the piRNA sensor transgene

mCherry expression stably repressed over generations after removal of the operon (OFF) transgene 